\title{
Fixed Cellular Rural Networks in Developing Countries: A Performance Evaluation
}

\author{
DINESH K. ANVEKAR ${ }^{*}$, PRATHIMA AGRAWAL ${ }^{+}$, and TEJASKUMAR PATEL ${ }^{*}$ \\ * Department of Electrical Communication Engineering \\ Indian Institute of Science, Bangalore 560012, India. \\ (E-mail: dka@ece.iisc.ernet.in) \\ + Networked Computing Research Department \\ AT\&T Bell Laboratories,600,Mountain Ave., Murray Hill, NJ 07974, USA
}

(E-mail: pa@research.att.com)

\begin{abstract}
Two variations of a generic architecture of fixed cellular rural network suitable for rural applications in developing countries are presented. Performance evaluation of the two architectures in terms of call blocking probability is carried out by using a simulation model of the networks. It is shown by simulation results that a combination of wired and wireless networks with channel borrowing for intervillage call traffic results in lower call blocking probability.
\end{abstract}

\section{Introduction}

In recent times there has been a rapid progress in providing telecommunication facilities in developing countries like India. However, a majority of rural areas are still without any basic communication facilities. There has been considerable effort made in India to ensure that there is atleast a phone in every village [1]. Any such effort is often impeded by the several difficulties for practical deployment of telecommunications technologies in developing countries. Some of the major deployment difficulties are : high initial capital costs, incompatibility of equipment, obsolescence of affordable equipment, different service quality requirement, and inadequate spectrum availability. Further, the telecormmunication equipment suitable for rural applications have to meet a different set of characteristics than those for use in urban areas. Considering their ease of deployment and cost-effectiveness for thin call traffic situations, wireless technologies appear to be an attractive choice for covering rural areas in a short time. However, a wireless network architecture for use in rural environment must meet the following characteristics: low start-up capital cost; flexibility;service quality equivalent to wired networks; expandability; optimum labour/material costs; and high spectrum utilization efficiency. On the other hand, the wireline technology is well proven and cost effective for higher subscriber densities. As has been shown in [2], wireline and wireless investment costs have a break even in the range of 20-40 subscribers per square kilometer. Therefore, considering the subscriber densities and the typical intra- and intervillage call traffic of a majority of villages, it appears that a telecommunication network architecture with wireline technology for intra-village calls and wireless technology for inter-village calls is a good choice for rapid deployment. Such an architecture can be gradually upgraded by replacing wireless sections with wireline technology. Keeping this in view, a comparative study of two telecommunication network architectures with respect to their call blocking performance was carried out. Some of the major aspects of this work are presented in the following Sections.

\section{Rural Network Architectures}

A generic architecture for a rural telecommunication network with fixed macrocell is shown in Fig. 1. Here, several neighbouring villages are grouped to form a macrocell which has a single base station (BS). The base stations are linked through high-speed wireline or wireless trunks or through links to a satellite. The subscribers within a village are connected to a remote switching unit (RSU) which manages all the intra-village call traffic. The RSUs are linked to the base station through wireless links. With this architecture, as the macrocells are often widely separated from each other, and the coverages of the BSs and RSUs do not overlap, a high degree of frequency reuse is possible leading to high spectrum utilization efficiency.

The two network architectures (NAs) considered in this work are shown in Figs 2 and 3 . In the architecture NA1, shown in Fig. 2, the village subscribers (VSs) are con- 
Table 1: Comparison of fixed cellular network architectures

\begin{tabular}{|l|c|c|}
\hline \hline & Architecture NA1 & Architecture NA2 \\
\hline Deployment Difficulty & High & Low \\
\hline Complexity of telephone sets & Low & High \\
\hline Grade of service for intra-village call traffic & Very Good & Fair \\
\hline Overall grade of service & Good & Fair \\
\hline Flexibility & Poor & Good \\
\hline Cochannel interference & Low & Medium \\
\hline Complexity of RSUs & Medium & High \\
\hline \hline
\end{tabular}

nected through traditional wired links. The subscriber equipment is just the conventional telephone set which is powered from a central battery in the RSU. An advantage of this architecture is that intra-village call traffic does not use up any radio spectrum. Also, intra-village calls are not blocked. However, inter-village and long distance calls still require a wireless channel between the RSU and the BS.

The network architecture NA2, shown in Fig. 3, has wireless links both between BS and RSUs, and between RSUs and VSs. This architecture has the advantage of ease of deployment but has the limitations of higher complexity and cost of subscriber equipment, higher call blocking rates and lower grade of service for inter-village calls when intra-village call traffic is high. As the telephone sets in this architecture are to be powered locally their maintenance costs are higher, and if powered from a. c. power source, the telephone service is affected during power failures.

A comparison of the two architectures is given in Table 1. From this Table it is clear that the architecture NA1 is superior to the architecture NA2 for deployment in a rural environment. A detailed quantitive evaluation of the performance and costs for the two architectures is necessary for making a choice in any given village environment. In this work, a simulation based performance evaluation in terms of call blocking probability (CBP) is carried out. Relevant aspects of the simulation study are given in the following Sections.

\section{Simulation Model}

A schematic representation of the simulation model used for evaluating CBPs for the two architectures is shown in Fig. 4. A macrocell with four villages is considered. The number of wireless channels in village $\mathrm{i}$ is $\mathrm{n}_{l i}$ and the number of channels between its RSU and the BS is $\mathrm{n}_{r i}$. The call traffic in each village is assumed to follow the Poisson distribution, and call holding time for each call is assumed to be exponentially distributed. In the simulation program, the call traffic for all the villages is generated by a single process at a rate which is four times the call arrival rate in each village. These calls are then distributed with equal probability over the four villages. The total offered traffic within a village $a_{o i}$ consists of the intra-village or local traffic $a_{l i}$ and inter-village or remote traffic $a_{r i}$. The fraction $f r c=a_{r i} / a_{o i}$ is a parameter used for the different simulation runs.

When a new call is generated for a village, a binomial distribution function with 'frc' as the variable is used to determine whether it is a local call or a remote call. Every local call needs two local channels, and a remote call needs four channels (one in the source village, one in the destination village, and two between the BS and the RSUs). For simplicity in this work, it is assumed that $\mathrm{n}_{l i}=\mathrm{n}_{l}(\mathrm{i}=1-4)$, and $\mathrm{n}_{r i}=\mathrm{n}_{r}(\mathrm{i}=1-4)$. While the number of channels between each RSU and the BS is thus assumed to be fixed as $\mathrm{n}_{r}$, it is allowed to be dynamically enhanced by borrowing channels from the neighbouring RSUs. However, the maximum number of channels $\left(\mathrm{n}_{r \max }\right)$ between an RSU and the BS allowed at any time is kept fixed for the different simulation runs.

The above simulation model was implemented using the Simscript II language, and CBPs for the two architectures were evaluated for different offered traffic and simulation parameters. The results of the simulations are discussed in the next Section.

\section{Simulation Results}

The input parameters used for the simulations are given in Table 2, and Figures 5,6 and 7 show the CBPs for the two architectures for different offered traffic. For the architecture NA2, as expected and seen in Fig. 5, CBP 
Table 2: Simulation input parameters

\begin{tabular}{|l|l|}
\hline \hline Number of villages & 4 \\
\hline Number of local channels/village $\left(\mathrm{n}_{l}\right)$ & 16 \\
\hline $\begin{array}{l}\text { Number of remote channels/village } \\
\left(\mathrm{n}_{r}\right)\end{array}$ & 5 \\
\hline $\begin{array}{l}\text { Maximum } \mathrm{n}_{r} \text { with channel borrow- } \\
\text { ing }\left(\mathrm{n}_{r m a x}\right)\end{array}$ & 8 \\
\hline Mean call holding time & $60 \mathrm{~s}$ \\
\hline Number of calls to be simulated & 100000 \\
\hline $\begin{array}{l}\text { Number of calls in the beginning } \\
\text { after which statistics are to be } \\
\text { collected }\end{array}$ & 5000 \\
\hline \hline
\end{tabular}

for local call traffic increases with the increase in offered traffic. It is to be noted that for the architecture NA1, as the local calls are through dedicated per-subscriber wirelines, the CBP for local call traffic is zero.

Fig. 6 shows CBP for remote call traffic. As seen here, NA1 has much lower CBP than NA2. The overall CBP (for both local and remote calls) for NA1 is also much lower than that for NA2 as clear from Fig. 7.

The variation of CBP with the fraction 'frc' is shown in Fig. 8. For this and the following simulation runs the value of $n_{\text {rmax }}$ was set as 10 . The architecture NA1 has much lower CBP for lower values of 'frc' but its CBP approaches that of NA2 for higher 'frc'. This shows that NA1 is a better choice for rural traffic with low 'frc'.

The effect of increasing $\mathrm{n}_{l}$ on CBP is shown in Fig. 9. As expected, increasing $n_{l}$ has no effect on CBP for the architecture NA1, but for NA2, CBP decreases with $n_{l}$. Also, to achieve the same call blocking performance as NA1, the architecture NA2 needs more number of local channels per village.

Fig. 10 shows the variation of CBP with $\mathrm{n}_{r}$. The decrease of CBP with increase in $n_{r}$ is as expected. However, the flat CBP curve for NA2 shows that $\mathrm{n}_{l}$ is a restricting factor for this case.

A comparison of CBP for the two architectures for cases with and without channel borrowing is provided by the results shown in Fig. 11. As seen here, the improvement in performance with channel borrowing is higher for NA1 than for NA2.

The above simulation results lead to the following observations:

(i) The architecture NA1 has much lower CBP, espe- cially with lower remote call traffic

(ii) The architecture NA2 has generally higher CBP as compared to the architecture NA1

(iii) CBP for remote traffic is improved by allowing borrowing of BS-RSU channels.

\section{Conclusion}

A simulation study of performance in terms of call blocking probability of two fixed cellular network architectures with wireless or wireline local coverage has been presented in this paper. The simulation results have shown that a rural fixed macrocellular architecture with a wired intravillage network and a wireless inter-village network is a preferable solution for rural applications in developing countries. Performance evaluation of the architectures with reference to other performance criteria is necessary for making a good choice for any given rural communication scenerio.

\section{References}

[1] B.S.Murthy," A Phone in Every Village," Telecommunication Research Centre, Dept. of Telecommunications, India, 1987

[2] H.Mannisto and K. Tuisku," Cost Comparison of Wireline and Wireless Access for Public Switched Telephone Network (PSTN)," Proceedings of IEEE International Conference on Personal Wireless Communications, Bangalore, India, pp. 37-39, Aug. 1994 